\title{
SVM-RBF Parameters Testing Optimization Using Cross Validation and Grid Search to Improve Multiclass Classification
}

\author{
F. Budiman ${ }^{1}$ \\ Department of Computer Science, University of Dian Nuswantoro, \\ Semarang, Indonesia \\ 1 ORCID: oooo-0oo2-8552-6778, fikri.budiman@dsn.dinus.ac.id
}

\begin{abstract}
The accuracy of using optimal parameter values in kernel functions is as a determinant to obtain maximum accuracy results on Image retrieval with Support Vector Machine (SVM) classification. Experiments conducted in this study aimed to obtain optimal Gaussian / Radial Basis Function (RBF) kernel function parameter values on non-linear multi class Support Vector Machine (SVM) method. Cross Validation and Grid Search methods were applied in analyzing and testing the optimization range of SVM-RBF kernel parameter values to recognize the image of Indonesian traditional Batik which has geometric decorative patterns. In addition, a feature dataset of Batik images from the results of Discrete Wavelet Transform (DWT) level $3 \mathrm{db} 2$ was used in this study. The feature dataset was used as training and test dataset. By using Cross validation and Grid Search, it resulted in the range value of parameter $\mathrm{C}=\left\{2^{6.5}, 2^{6.75}, 2^{7}, 2^{7.25}, 2^{7 \cdot 5}, 2^{7 \cdot 75}, 2^{8}\right\}$ and $\gamma=\left\{2^{-14.5}, 2^{-14.75}, 2^{-15}, 2^{-15.25}, 2^{-15.5}, 2^{-15.75}, 2^{-16}\right\}$, and the accuracy value of maximum classification for parameter $C=2^{7}$ and $\gamma=2^{-15}$. These range results of parameter values and optimal parameter values can be used as a reference in applying parameters on image recognition with geometric decorative motif texture using SVM-RBF kernel classification.
\end{abstract}

Keywords: Cross Validation, Geometric Decorative Motif, Grid Search, Radial Basis Function, Support Vector Machine.

\section{Introduction}

Evaluation to optimize the use of Gaussian SVM-RBF kernel parameters is necessarily significant to do. It is conducted to get the results of multi class classification with minimum (smallest) error. Multi class classification optimization with non-linear SVM depends very much on its kernel and parameters functions. Moreover, Gaussian RBF kernel is recommended to obtain maximum non-linear SVM classification results for a new dataset [1]-[4]. This is due to fact that it has the same performance as the linear kernel in parameter cost (C) and gamma $(\gamma)$ / sigma $(\sigma)$ with a certain value in classification optimization. Parameter estimation is needed in the form of constant parameter values for soft margins (C) and kernel parameters $(\gamma)$ to obtain the results of new test dataset classification with a maximum nonlinear Gaussian SVM-RBF kernel [3], [4]. Parameters C and $\gamma$ with right values can keep the bias (measure of error contribution) and variance (measure of deviations) low when it is used in different training datasets by Cross Validation method. In maximizing the result of non-linear SVM multi-class classification by Gaussian RBF kernel, it is necessary to evaluate the use of geometric decorative motif datasets for training and testing. Grid Search method with Cross Validation can be used to evaluate the success in estimating RBF kernel parameters. In addition, Grid Search is a search model for the right RBF kernel parameter values by testing parameter values in a certain interval [2]. Each value in the interval is tested and the 
following value tested is the exponential addition of the parameter value. After finding the best parameter value, then another test is conducted at a smaller range among the best parameters. In short, this paper discusses the evaluation of parameter testing using the Grid Search method with Cross Validation which is implemented on the Optimize Accuracy value of Indonesian Batik Images Classification Research [5].

Training and test datasets used in this experiment are four classes of geometric decorative ornaments on the texture of Indonesian batik motifs (figure 1). The use of this batik dataset is due to the fact that each class has the same geometric decorative motifs with very diverse motif patterns. This motive patterns diversity will eventually lead to a high complexity in the separation between their classes, because classes separation in classification function (hyperplane) cannot be done linearly (non-linear). The effort to increase accurationvalue of nonlinear multiclasses classification with a dataset of transient batik images is necessary to determine kernel parameters. However, it has not been conducted in some previous studies. In additition, it is necessary to do parameter determination since it can produce a good new feature (high dimension). Thus, this experiment results in a maximum hyperplane to be applied to image dataset with geometric decorative motifs. Moreover, a limited dataset in this study makes it necessary to do an experiment to determine new parameter value on the nonlinear multi-classes classification of SVM-RBF kernels with Grid Search and Cross Validation method to obtain maximum accuracy value.

Geometric decoration is an abstract ornamental motif in the form of circles, rectangles, curved lines, zigzags, and/or triangles as found in the decorative motifs of Batik images (figure 1). Indonesia Batik image is the work of fine art produced on a piece of white fabric textile, i.e. it decorates the textile surface by holding the dye. Batik is an Indonesian heritage showing the intelligence of the ancestors in creating beauty in a piece of cloth. Color retaining on the textile surface is done by applying wax liquid (wax) using a traditional tool called "writing canthing (canting tulis)" and "stamp canthing (canthing cap)" as shown in Figure 2. The color retention process is known as wax-resist dyeing process [6], [7]. Indonesian batik has been recognized by UNESCO since 2009 as a "Representative List of the Intangible Cultural Heritage of Humanity"

There are 80 images in training and test datasets which are divided into 4 classes. Class 1 consists of 20 images; class 2 is 16 images; class 3 is 19 images, and class 4 is 25 images. Figure 1 shows the examples of class differentiation based on batik motifs which vary greatly. Batik image feature used is the result of feature extraction with Discrete Wavelet Transform (DWT) level 3 and the coefficient of Daubechies 2 (db2) scaling function. Furthermore, the use of DWT level $3 \mathrm{db} 2$ is the best result from the comparison of decomposition level and type of scaling function coefficient in previous [8]. DWT-2D is the most effective method to apply on the texture of geometric decorative motifs of traditional batik images. DWT can produce good features for images possessing multi-resolution space and with varying image scale transformation, and also can produce features by distinguishing image intensity in sub-band spaces [9].
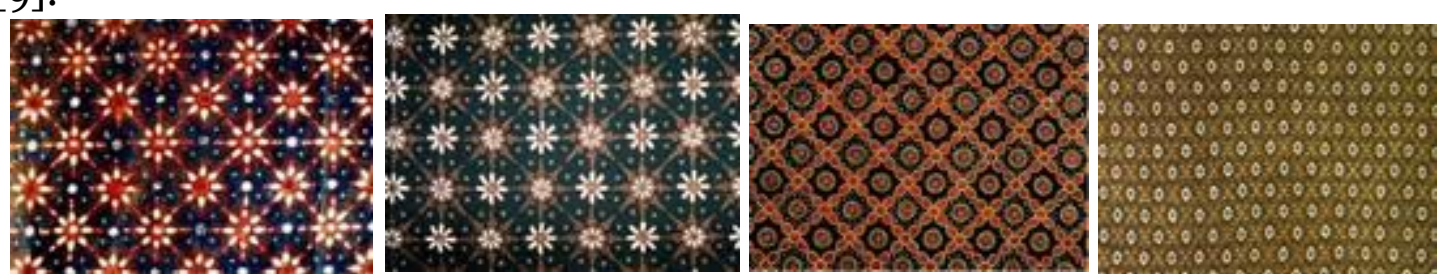

Class 1: "Ceplok" motif; Note: ceplok (circle-shaped)
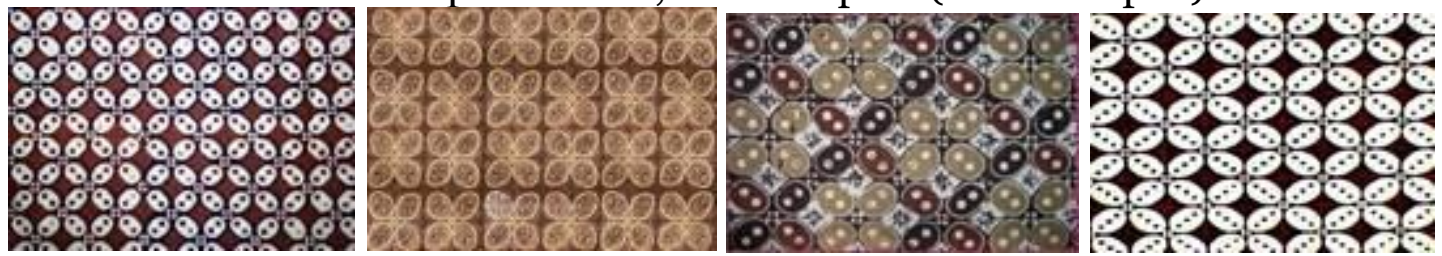

Class 2 : "Kawung" motif; Note: kawung (javanese : sugar palm fruit motif with 4 corners) 

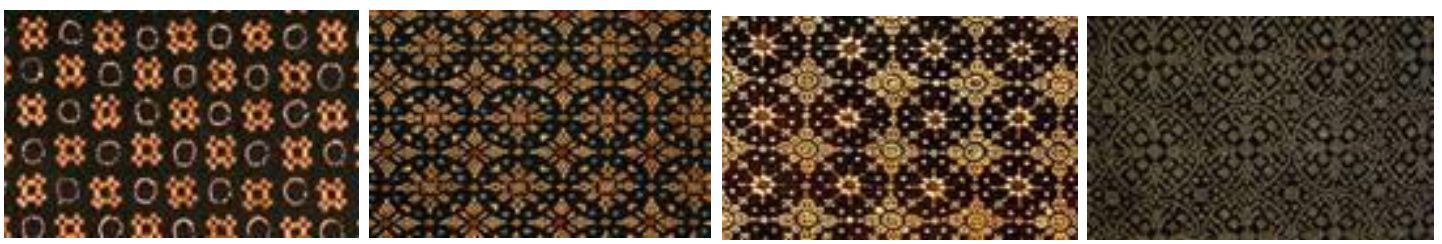

Class 3 : "Nitik" motif; Note: nitik (dot; tiny square dot)
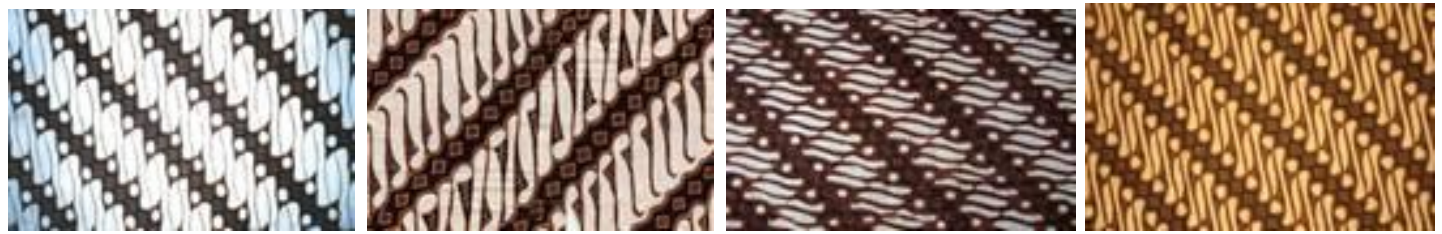

Class 4 : "Parang"; Note: parang (big knife, longer than knife but shorter than sword)

Figure 1. Batik Motif Class (f. Budiman, et al. 2017)

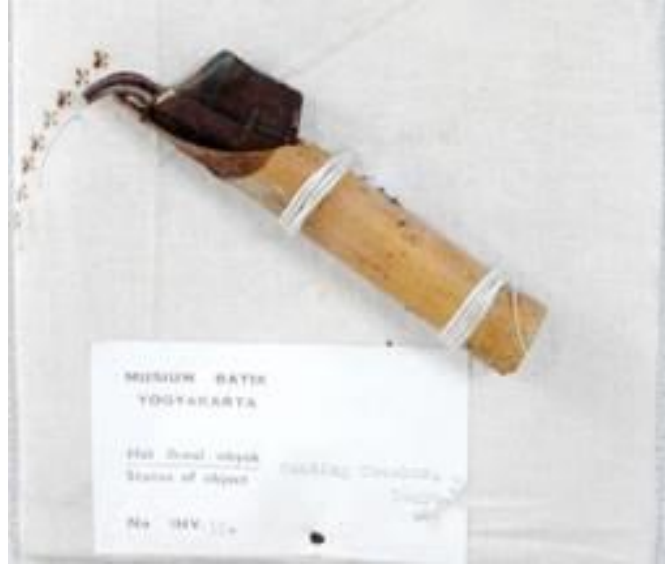

(a)

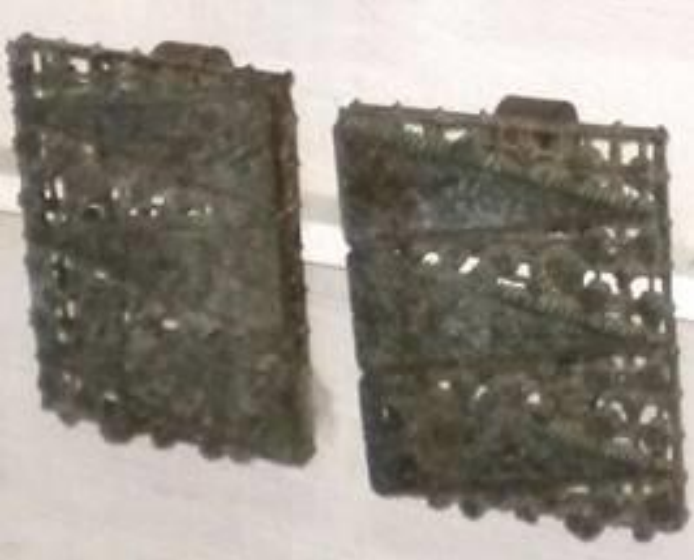

(b)

Figure 2. Tools to create Batik : a. "canting"; b. "stamp/cap"

\section{Research Method}

The accuracy of classification method resulted from training process with SVM relies heavily on kernel functions and the selection of parameter values used. Then, SVM classification optimization for non-linear multi class cases is conducted in the next test phase analysis by the use of Gaussian / Radial Basis Function (RBF) kernel parameters. Optimal RBF parameter value highly depends on the dataset used. Optimization of non-linear multi-class SVM-RBF classification results with Grid Search and Cross Validation processes is done to assure the minimization of over and under fittings and to obtain a combination of kernel RBF parameter values on space parameters which produce maximum classification accuracy values. Parameters $\mathrm{C}$ and $\gamma\left(1 / 2^{\sigma_{2}}\right)$ with the right values can maintain the bias (a measure of the error contribution) and the variance (measure of the deviations) remains low when using a different training dataset with the $v$-fold Cross Validation method.

Table 1 shows high $\mathrm{C}$ value and low $\gamma$ value which cause over fitting. Conversely, low $\mathrm{C}$ value and high $\gamma$ value will lead to under fitting [10]. The smaller $\mathrm{C}$ value, the more it will ignore the feature point as a support vector existing close to the hyperplane; as well as increase the maximum margin (figure 3). The higher Gamma value $(\gamma)$ will increase the vector support area increases and the flexibility of the decision boundary (hyperplane). Thus, support vector will not cause a broad influence (figure 4). 
Table 1. The Influence of $\mathrm{C}$ and $\gamma$ Values

\begin{tabular}{|l|l|l|}
\hline & Low Variance & High Variance \\
\hline Low Bias & $\begin{array}{l}\text { Right } \gamma \text { value ? } \\
\text { Right C value? }\end{array}$ & $\begin{array}{l}\text { Low } \gamma \text { value } \\
\text { High C value }\end{array}$ \\
\hline High Bias & $\begin{array}{l}\text { High } \gamma \text { value } \\
\text { Low C value }\end{array}$ & $\begin{array}{l}\text { high bias } \rightarrow \text { under fitting } \\
\text { high variance } \rightarrow \text { over fitting }\end{array}$ \\
\hline
\end{tabular}
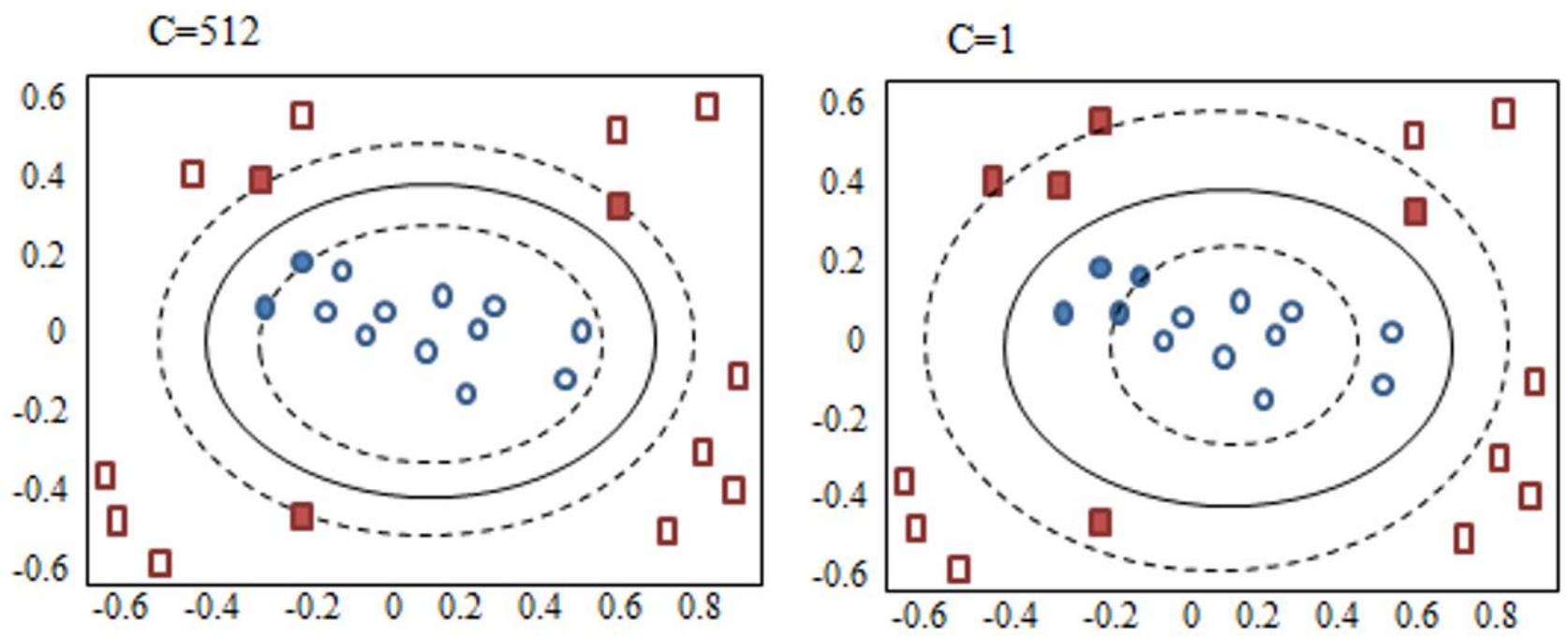

Figure 3. SVM-RBF Kernel Classification for $\mathrm{C}=512$ and $\mathrm{C}=1$,

$\square \square=$ feature vector class $+1, \quad 0 \circ \quad=$ feature vector class -1 ,

$\square$ = support vector
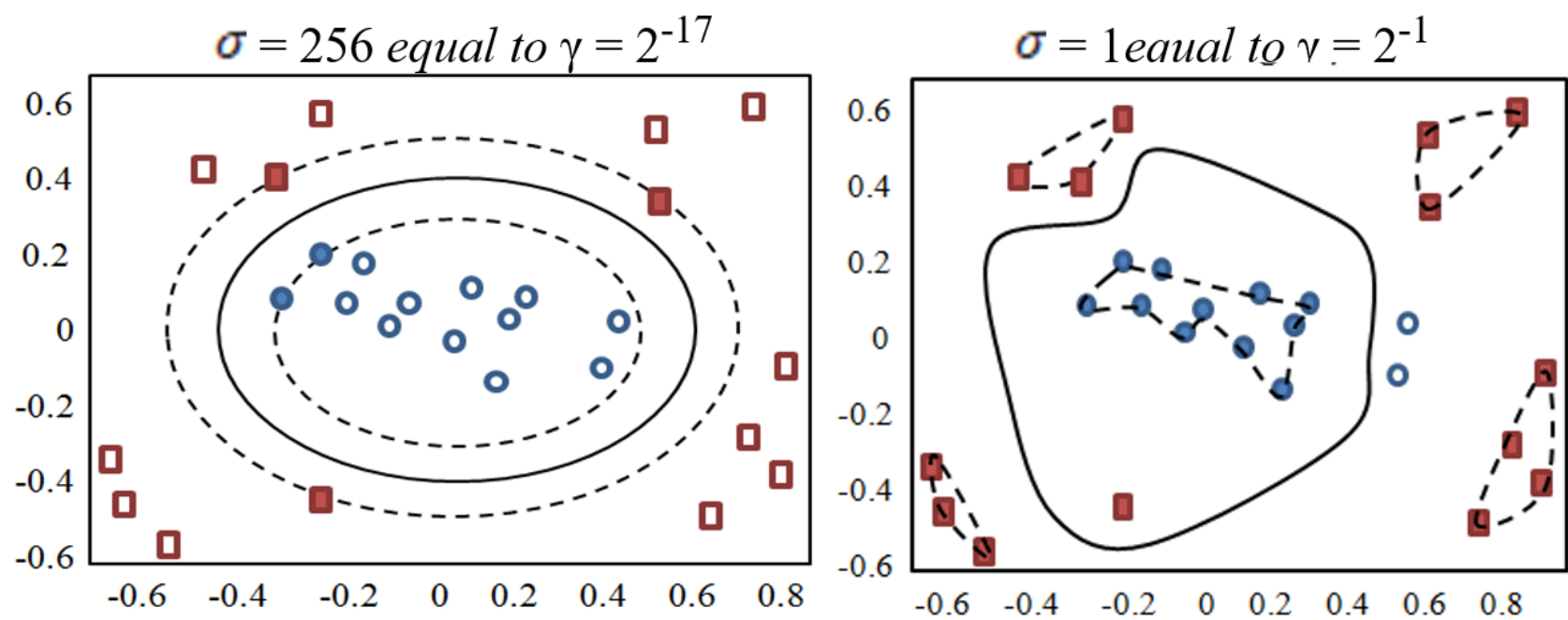

Figure 4. SVM-RBF Kernel Classification for $\gamma=2^{-1}$ and $\gamma=2^{-17}$,

$\square \square=$ feature vector class $+1, \quad 0 \circ=$ feature vector class -1 ,

$\square \circ$ = support vector

Several different test datasets required test datasets testing to obtain the accuracy of classification results with minimum error rate. Problems occur if the test of SVM-RBF parameters on the training dataset has obtained good accuracy on the test dataset since it will lead to a question whether it will also result in a good accuracy when used on different datasets. To test different datasets in a limited number of datasets, Cross Validation (CV) method can be applied in Grid Search process [2], [11]. CV method divides the dataset into v partitions ( $v$-fold) randomly, each partition has an index number 1 through v. Futhermore, commonly used Par- 
titions are divided into 10 partitions or 10-fold Cross Validation[9], [12], [13]. For 10 partitions, the test is conducted 10 times by leave-one-out method, i.e. one part is used interchangeably into test dataset and the other dataset $(\mathrm{v}-1)$ is used for training dataset as shown in table 2.

Table 2. 10-fold Cross Validation

\begin{tabular}{|l|l|l|}
\hline $\begin{array}{l}\text { Testing } \\
\text { No }\end{array}$ & Training Dataset & $\begin{array}{l}\text { Test } \\
\text { Dataset }\end{array}$ \\
\hline 1 & v1+v2+v3+v4+v5+v6+v7+v8+v9 & v10 \\
\hline 2 & v1+v2+v3+v4+v5+v6+v7+v8+v10 & v9 \\
\hline 3 & v1+v2+v3+v4+v5+v6+v7+v9+v10 & v8 \\
\hline 4 & v1+v2+v3+v4+v5+v6+v8+v9+v10 & v7 \\
\hline 5 & v1+v2+v3+v4+v5+v7+v8+v9+v10 & v6 \\
\hline 6 & v1+v2+v3+v4+v6+v7+v8+v9+v10 & v5 \\
\hline 7 & v1+v2+v3+v5+v6+v7+v8+v9+v10 & v4 \\
\hline 8 & v1+v2+v4+v5+v6+v7+v8+v9+v10 & v3 \\
\hline 9 & v1+v3+v4+v5+v6+v7+v8+v9+v10 & v2 \\
\hline 10 & v2+v3+v4+v5+v6+v7+v8+v9+v10 & v1 \\
\hline
\end{tabular}

In every test, parameter $\mathrm{C}$ and $\gamma$ combination is carried out 10 times classification with 10 different training and test datasets $(10$-fold $)$. This is to ensure that there is no excessive overfitting on the test with different testing data. Each use of parameter $\mathrm{C}$ and $\gamma$ combination was tested 10 times. In addition, a selection of random and balanced training and test datasets is conducted in each test for class 1, 2, 3 and 4 on feature vector file consisting of 80 batik image records. Training and testing using 80 traditional batik images as well as 10 -fold $\mathrm{CV}$ method with leave-one-out process on random image feature vector selection only produces 8 feature vectors for every test dataset used in each test. Since the result with these 8 feature vectors is still too small for the test dataset, random selection is then carried out by doing hold out process using 30\% percentage range for test dataset and the rest is for training dataset in increasing the number of test datasets in this study.

Hold out process, i.e. randomly selecting records for test data, is conducted on CV for feature vector files containing 80 batik image records. Class 1 ("ceplok" motif) of 1-20 records resulted in selected 6 records, class 2 ("kawung" motif) record 21-36 selected 4 records, class 3 ("nitik" motif) record 37-55 selected 5 records, and class 4 ("parang" motif) selected 7 records. Random selection is conducted in each test of parameter $\mathrm{C}$ and $\gamma$ combination. Moreover, the results of hold out application to 80 image feature vectors can be seen in table 3. Consequently, table 3 can describe 10 classifications in which every process of training and testing is different, each training dataset contains 58 image feature vectors and every test dataset consists of 22 image feature vectors. Feature vector column describes training and test classification 1st to 10th and index number ' 1 ' show that the record is for training data, while index ' $\mathrm{O}$ ' shows that the record is test data. 
Table 3. Result of Cross Validation

\begin{tabular}{|c|c|c|c|c|c|c|c|c|c|c|c|}
\hline \multirow{2}{*}{$\begin{array}{l}\text { Record } \\
\text { Number }\end{array}$} & \multirow[t]{2}{*}{ Class } & \multicolumn{10}{|c|}{$\begin{array}{c}\text { Feature Vector of training and } \\
\text { test classification No }\end{array}$} \\
\hline & & 1 & 2 & 3 & 4 & 5 & 6 & 7 & 8 & 9 & 10 \\
\hline 1 & 1 & 1 & 1 & 1 & 1 & 1 & $\mathrm{O}$ & $\mathrm{O}$ & 1 & 1 & 1 \\
\hline 2 & 1 & 0 & 1 & 1 & 1 & 0 & 1 & 0 & 1 & 1 & 1 \\
\hline 3 & 1 & 1 & $\mathrm{O}$ & $\mathrm{O}$ & 1 & 1 & $\mathrm{O}$ & 1 & 1 & $\mathrm{O}$ & 1 \\
\hline 4 & 1 & 1 & 1 & $\mathrm{O}$ & 1 & $\mathrm{O}$ & 1 & $\mathrm{O}$ & 1 & 1 & 1 \\
\hline 5 & 1 & $\mathrm{O}$ & $\mathrm{O}$ & 1 & $\mathrm{O}$ & 1 & 1 & 1 & 1 & 1 & 1 \\
\hline$\ldots$ & $\ldots$ & $\ldots$ & $\ldots$ & $\ldots$ & $\ldots$ & $\ldots$ & $\ldots$ & $\ldots$ & $\ldots$ & $\ldots$ & $\ldots$. \\
\hline$\ldots$ & $\ldots$ & $\ldots$ & $\ldots$ & $\ldots$ & $\ldots$ & $\ldots$ & $\ldots$ & $\ldots$ & $\ldots$ & $\ldots$ & $\ldots$. \\
\hline 78 & 4 & $\mathrm{O}$ & $\mathrm{O}$ & 1 & 1 & 1 & 1 & $\mathrm{O}$ & 1 & $\mathrm{O}$ & $\mathrm{O}$ \\
\hline 79 & 4 & 1 & 1 & 1 & 1 & 1 & 0 & $\mathrm{O}$ & $\mathrm{O}$ & 1 & 1 \\
\hline 80 & 4 & 1 & 1 & 1 & 1 & 1 & 1 & 1 & O & 1 & 1 \\
\hline
\end{tabular}

Optimizing the estimation of the best parameter values in this experiment uses a range of parameter values. Besides, searching using this values range is commonly called Grid Search method. Initial trial of Grid Search method is done using a wide range of RBF, C and $\gamma$ kernel parameter values. Then, after finding the best parameters, testing with a narrower range of values is conducted to obtain parameter values which will result in the best classification accuracy value. Actually there is no provision for the right range for Grid Search method. In this case, the wider the parameters range is, the more effective it is to get the best parameters $\mathrm{C}$ and $\gamma$ combination as to significantly increase the accuracy value in image recognition classification. In the initial trial for value range estimation of parameter $\mathrm{RBF} C=\left\{2^{-3}, 2^{-1}, 2^{1}, 2^{3}\right.$, $\left.2^{5}, \ldots, 2^{13}, 2^{15}, 2^{17}\right\}$ and $\gamma=\left\{2^{-17}, 2^{-15}, 2^{-13}, \ldots, 2^{-5}, 2^{-3}, 2^{-1}, 2^{1}, 2^{3}\right\}$, ten classifications in each trial of parameters $\mathrm{C}$ and $\gamma$ combination are carried out using 10 training datasets and 10 test datasets from cross validation result with hold out process. Furthermore, there is no range requirement for $\mathrm{C}$ and $\gamma$ value estimation as test value of Gaussian RBF kernel parameter. The wider the range of values for these parameters is, the more effective the application of Grid Search parameter searching method with $v$-fold $C V$ will be tofind a combination of $\mathrm{C}$ and $\gamma\left(1 / 2 \sigma^{2}\right)$.

The best parameter combination for $\mathrm{C}$ and $\gamma$ values will produce maximum accuracy from the classification results. In previous experiments (Renukadevi and Thangaraj, 2013), the classification results with SVM-RBF kernel are still limited in the use of parameters combination by using 10 -fold Cross Validation and RBF kernel parameters to optimize constant value $\mathrm{C}=$ 0.125, and three values $\gamma(0.125,0.25$, and 0.75). Thus, it doesn't apply Grid Search. The range by adding exponentially different parameter values as in previous studies, $\mathrm{C}$ and $\gamma$ values range from 0.001 to 10,000 (Syarif, Prugel-Bernnett, and Adam, 2016); and C is between 1 and 1000 and $\sigma$ value is between 1 and 100 [12]. In Gaspar et al. [12] and Syarifet al. [13], parameter estimation optimization is not the goal of the studies. Instead, parameters are used to test the application of RBF, polynomial, and sigmoid kernel functions to obtain maximum accuracy values in SVM classification. Thus, there is no parameter optimization process in testing classification accuracy for certain datasets. 


\section{Results and Analysis}

The method used in this experiment initially results in a feature file of all images which are distributed into many classes using 3 levels decomposition and daubechies 2 Discrete Wavelet Transform (DWT) feature extractive method. This feature extraction method is used based on the writer's previous research [8]. Moreover, fold-cross validation (CV) with 10-fold CV is conducted to this feature file on each parameter value test. The use of 10-fold CV which is based on the results of 10-fold, 8-fold, 6-fold, 4-fold, and 2-fold tests on multi - class nonlinear SVM-RBF classification shows that the smaller the effect $k$ values is, the higher the error the image recognition is. This is due to the less training set is, the worse it is in representing hyperplane and margins for each class. In addition, the best parameter value estimation in this study is calculated using parameter value range as referred to Grid Search method. Then, each parameter value is tested 10 times with a dataset of different test feature files from 10 fold CV. This is conducted to ensure the parameters value used in the classification will lead to a relatively similar accuration value in each test.

One-against-all method of non-linear SVM classification with four classes consists of four non-linear binary class SVM classifications. Accordingly, parameter values $\mathrm{C}$ and $\gamma$ must be able to maximize classification results for four hyperplane formations and margins $(+1$ and 1). In the initial trial for value range estimation optimization of parameter $\mathrm{RBF} C=\left\{2^{-3}, 2^{-1}\right.$, $\left.2^{1}, 2^{3}, 2^{5}, \ldots, 2^{13}, 2^{15}, 2^{17}\right\}$ and $\gamma=\left\{2^{-17}, 2^{-15}, 2^{-13}, \ldots, 2^{-5}, 2^{-3}, 2^{-1}, 2^{1}, 2^{3}\right\}$, the first thing to do for standard parameter is like in the binary classification with value of $C=1$ and $\gamma=0.5$. Ten times classification using 10-fold CV in the initial trial for parameters combination $\mathrm{C}=1$ and $\gamma=0.5$ is done using 10 training datasets and 10 test datasets. In using RBF kernel parameter value $C=1 ; \gamma=0.5$ in SVM classification for test feature data with classes 1,2 and 3 which is yet well unrecognizable, the accuracy value is still low below 0.5 since it cannot produce $h y$ perplane and margins which are able to properly recognize the testing image for class 1,2 , and 3. High bias on the use of parameter $\mathrm{C}=1$ and $\gamma=0.5$ causes under fittings for class 1,2 and 3 , since the margin area generated from support vector does not have a broad influence in gathering a class. Besides, the result of classification accuracy value is still relatively the same when using values parameter combination of value $\gamma=\left\{2^{3}, 2^{1}, 2^{-1}\right\}$ and $C=\left\{2^{-3}, 2^{-1}\right.$, $2^{\circ}$. The use of parameter $C=2^{-1} ; \gamma=0.5, C=2^{-3} ; \gamma=0.5, C=1 ; \gamma=2^{1}$, and $C=1 ; \gamma=2^{3}$ provides a relatively similar accuracy value to $\mathrm{C}=1 ; \gamma=0.5$ which is still low (below 0.5 ). Consequently, it can be considered that there is still relatively high possibility of under fittingin class recognition from the test feature data. Furthermore, the greater $\mathrm{C}$ value is, the smaller the number of support vectorsis and the margin distance with the hyperplane will be much narrower. Thus, this can lead to over-fitting or misplacing classification class area to feature vectors near the hyperplane. Also, if the value of $\gamma$ is smaller, the support vectorwill be smaller. Then, the margin formed will have a smooth decision boundary that is likely similar to linear form.

Maximum recognition with low bias and low variance in the parameters range that have been determined in this study is obtained in parameter values $C=27$ and $\gamma=2^{-15}$ with the number of correctly recognized feature vectors is from 17 to 19 of 22 vector features of the test dataset in every test. Classification results and accuracy values are obtained from 10 tests with parameter values $\mathrm{C}=2^{7}$ and $\gamma=2^{-15}$ using different test datasets in each test. Moreover, the use of parameters $\mathrm{C} \geq 2^{9}$ and $\gamma \leq 2^{-17}$ leads to the increase in measure of deviations (variance) which cause an increase in over fitting in determining the class of test feature data. In consequence, the next test with Grid Search is carried out in a smaller range between $2^{5<} \mathrm{C}<$ $2^{9}$ and $2^{-17}<\gamma<2^{-15}$. Thus, the range used by Grid Search is $C=\left\{2^{6.5}, 2^{6.75}, \mathbf{2}^{7}, 2^{7.25}, 2^{7 \cdot 5}, 2^{7.75}\right.$, $\left.2^{8}\right\}$ and $\gamma=\left\{2^{-14.5}, 2^{-14.75}, \mathbf{2}^{-15}, 2^{-15.25}, 2^{-15.5}, 2^{-15 \cdot 75}, 2^{-16}\right\}$. Then a comparison with accuracy value on the use of parameters $\mathrm{C}=2^{7}$ and $\gamma=2^{-15}$ is conducted from this range.

Accuracy value of classification results with the use of parameter values $C=\left\{2^{6.50}, 2^{6.75}, 2^{7}\right.$, $\left.2^{7.25}, 2^{7.5}\right\}$ and $\gamma=2^{-15}$ (table 4 ) shows that parameter value $C$ is smaller than $2^{7}\left(2^{6.75}, 2^{6.5}\right)$. 
Accordingly, there is an increase of bias so that more under fitting occur which causes accuracy value in each classification trial tends to decrease in value (blue colored numbers/value). As seen in table 4 , the use of parameter value $C$ is higher than $2^{7}(27.25,27.5)$. Thus, there is a decrease in accuracy value (blue colored numbers) in each classification test. This happensdue to the fact that the increase in variance of $\mathrm{C}>2^{7}$ causes over fitting. The use of parameter values combination with $C=2^{7}$ and $\gamma=\left\{2^{-14.5}, 2^{-14.75}, 2^{-15}, 2^{-15.25}, 2^{-15.5}\right\}$ (table 5) results in a decrease in accuracy values (blue colored numbers) compared to the use of parameter values combination $27 ; \gamma=2^{-15}$ in every classification test with different test data. Parameter values $\gamma>2^{-15}\left(2^{-14.5}, 2^{-14.75}\right)$ show an increase in biasthat leads increasing misclassification due to under fitting, and parameter value $\gamma<2^{-15} \quad\left(2^{-15.25}, 2^{-15.5}\right)$ increases variance that creates more misclassification due to the occurrence of over fitting.

Table 4. Accuracy Value and The Use of Parameter Value $\mathrm{C}=\left\{\mathbf{2}^{6.50}, \mathbf{2}^{6.75}, \mathbf{2}^{7}, \mathbf{2}^{7.25}, \mathbf{2}^{7.5}\right\}$ and $\gamma=\mathbf{2}^{-\mathbf{1 5}}$

\begin{tabular}{|c|c|c|c|c|c|}
\hline \multirow{2}{*}{ Test } & $\begin{array}{c}\mathrm{C}=\mathbf{2}^{7} ; \\
\mathrm{N}=\mathbf{2}^{-15}\end{array}$ & $\begin{array}{c}\mathrm{C}=\mathbf{2}^{6.50} ; \\
\mathrm{\gamma}=\mathbf{2}^{-15}\end{array}$ & $\begin{array}{c}\mathrm{C}=\mathbf{2}^{6.75} \\
\mathrm{\gamma}=\mathbf{2}^{-15}\end{array}$ & $\begin{array}{c}\mathrm{C}=\mathbf{2}^{7.25} ; \\
\mathrm{\gamma}=\mathbf{2}^{-15}\end{array}$ & $\begin{array}{c}\mathrm{C}=\mathbf{2}^{7.50} ; \\
\mathrm{\gamma}=\mathbf{2}^{-15}\end{array}$ \\
\cline { 2 - 6 } & \multicolumn{5}{|c|}{ Accuracy Value } \\
\hline 1 & 0.864 & 0.818 & 0.864 & 0.864 & 0.818 \\
\hline 2 & 0.773 & 0.727 & 0.773 & 0.727 & 0.773 \\
\hline 3 & 0.864 & 0.773 & 0.818 & 0.864 & 0.773 \\
\hline 4 & 0.864 & 0.864 & 0.864 & 0.818 & 0.864 \\
\hline 5 & 0.773 & 0.773 & 0.727 & 0.773 & 0.773 \\
\hline 6 & 0.818 & 0.773 & 0.818 & 0.818 & 0.818 \\
\hline 7 & 0.818 & 0.682 & 0.818 & 0.773 & 0.727 \\
\hline 8 & 0.864 & 0.864 & $\mathbf{0 . 9 0 9}$ & $\mathbf{0 . 9 0 9}$ & 0.864 \\
\hline 9 & 0.773 & 0.682 & 0.773 & 0.727 & 0.682 \\
\hline 10 & 0.773 & 0.727 & 0.773 & 0.682 & 0.727 \\
\hline
\end{tabular}

Table 5. Accuracy Value and The Use of Parameter Value $\mathrm{C}=\mathbf{2}^{7}$ and $\gamma=\left\{2^{-14.5}, 2^{-14.75}, \mathbf{2}^{-15}, 2^{-15.25}, 2^{-15.5}\right\}$

\begin{tabular}{|c|c|c|c|c|c|}
\hline \multirow{2}{*}{$\begin{array}{c}\text { Test } \\
\text { No }\end{array}$} & $\mathbf{C}=\mathbf{2}^{7} ; \gamma=\mathbf{2}^{-15}$ & $\mathbf{C}=\mathbf{2}^{7} ; \gamma=\mathbf{2}^{-14.5}$ & $\mathbf{C}=\mathbf{2}^{7} ; \gamma=\mathbf{2}^{-14.75}$ & $\mathbf{C}=\mathbf{2}^{7} ; \gamma=\mathbf{2}^{-15.25}$ & $\mathbf{C}=\mathbf{2}^{7} ; \gamma=2^{-15.5}$ \\
\hline 1 & 0.864 & 0.727 & 0.818 & 0.818 & 0.773 \\
\hline 2 & 0.773 & 0.773 & 0.727 & 0.727 & 0.727 \\
\hline 3 & 0.864 & 0.818 & 0.727 & 0.818 & 0.773 \\
\hline 4 & 0.864 & 0.727 & 0.7727 & 0.818 & 0.773 \\
\hline 5 & 0.773 & 0.727 & 0.773 & 0.727 & 0.773 \\
\hline 6 & 0.818 & 0.773 & 0.818 & 0.773 & 0.727 \\
\hline 7 & 0.818 & 0.727 & 0.727 & 0.727 & 0.818 \\
\hline 8 & 0.864 & $\mathbf{0 . 9 0 9}$ & $\mathbf{0 . 9 0 9}$ & 0.818 & 0.864 \\
\hline 9 & 0.773 & 0.682 & 0.727 & 0.773 & 0.773 \\
\hline 10 & 0.773 & 0.727 & 0.727 & 0.682 & 0.727 \\
\hline
\end{tabular}




\section{Conclusion}

Optimization of RBF kernel parameters has been carried out in this experiment to get maximum accuracy value in non-linear multi-class SVM classification method to recognize images with geometric decorative motifs. Optimization by using Grid Search method and 10-fold Cross Validation with holdout generates 10 test datasets and 10 training datasets from randomly selected feature vectors. With 10 tests for each combination of $\mathrm{C}$ and $\gamma$, and with different test datasets for each test, it can obtain the smallest range of parameter combinations $\mathrm{C}$ and $\gamma$ with low bias and low variance which produces the highest classification accuracy value.

The best from the method of determining optimal parameter values of space $C=\left\{2^{6.5}, 2^{6.75}\right.$, $\left.2^{7}, 2^{7 \cdot 25}, 2^{7 \cdot 5}, 2^{7 \cdot 75}, 2^{8}\right\}$ and $\gamma=\left\{2^{-14.5}, 2^{-14.75}, 2^{-15}, 2^{-15.25}, 2^{-15.5}, 2^{-15.75}, 2^{-16}\right\}$ with parameters combination $\mathrm{C}=2^{7}$ and $\gamma=2^{-15}$. Moreover, it is obtained using Grid Search testing method and Cross Validation with hold out process which uses 30\% percentage for the test dataset and the rest for the training dataset. Parameters combination $\mathrm{C}=27$ and $\gamma=2-15$ is used to evaluate the accuracy of optimal value determining method in this study. This is to ensure that there is no high level of accuracy difference in the use of different training and testing image datasets with different number of test datasets.

In trial analysis, the use of parameters combination with ranges of $C=\left\{2^{6.5}, 2^{6.75}, 2^{7}, 2^{7.25}, 2^{7 \cdot 5}\right.$, $\left.2^{7 \cdot 75}, 2^{8}\right\}$ and $\gamma=\left\{2^{-14.5}, 2^{-14.75}, 2^{-15}, 2^{-15.25}, 2^{-15.5}, 2^{-15 \cdot 75}, 2^{-16}\right\}$ shows no significant changes. Moreover, the best results remain in the combination of parameter $C=2^{7}$ and $\gamma=2^{-15}$. Based on the results of this study, it indicates that to get the accuracy value in the identification and/or recognition of traditional batik with textures possessing geometric decorative motifs with multi-scale patterns and multi-color resolution; parameter values are needed to optimize the performance of SVM-RBF kernel classification. In addition,these maximum parameter value and smaller range of parameter values can be used as a reference for digital image recognition with textures possessing geometric decorative motifs using SVM-RBF kernel classification.

\section{References}

1. Hofmann, Martin. "Support Vector Machine-Kernel and The Kernel Trick", Bamberg University, 2006.

2. Hsu, Chih-Wei., Chang, Chih-Chung., Lin Chih-Jen., "A Practice Guide to Support Vector Classification”. Department of Computer Science, National Taiwan University, 2010.

3. Renukadevi, N,T., Thangaraj, P., "Performance Evaluation Of Svm-Rbf Kernel For Medical Image Classification”, Global Journal of Computer Science and Technology Graphics \& Vision, vol.13 issue 4, Global Journal Inc, USA, 2013.

4. Rosales-Perez, Alejandro., Escalante, Hugo Jair., Gonzales, Jesus A., Reyes-Garcia, Carlos A., "Bias And Variance Optimization For Svms Model Selection", Procedings of the Twenty-Sixth International Florida Artificial Intelligence Research Society Conference, 2013.

5. Budiman, F., Suhendra, A., Agushinta, D., \&Tarigan, A, "Determination Of SVM-RBF Kernel Space Parameter To Optimize Accuracy Value Of Indonesian Batik Images Classification”, Journal of Computer Science. 13(11):590-599, 2017.

6. Riyanto, Rahayu, Slamet, and Pamungkas, Wisnu, "Handbook of Indonesian Batik". The Institute For Research and Development of Handicraft and Batik Industrie, Yogyakarta, 1997.

7. Tirta, Iwan, "BATIK SebuahLakon”, Gaya Favorit Press, Jakarta, 2009.

8. Budiman, F., Suhendra, A., Agushinta, D., \&Tarigan, A.,"Wavelet Decomposition Levels Analysis For Indonesia Traditional Batik Classification", Journal of Theoretical \& Applied Information Technology, 92(2):389-394, 2016.

9. Virmani,Jitendra.,Kumar,Vinod.,Karla,Naveen.,Khandelwal,Niranjan., "SVM-Based Characterization Of Liver Ultrasound Images Using Wavelet Packet Texture Descriptors", Journal of Digital Imaging (2013)26:530-543, Springer, 2013. 
10. Boser, B., Guyan, I., Vapnik, V., "A Training Algorithm for Optimal Margin Classfiers, Fifth Annual Workshop on Computational Learning Theory”, New York:ACM Press, 1992.

11. Tsamardinos, Ioannis, Rakhshani, Amin, danLagani,Vinceszo, "Performance-Estimation Properties Of Cross Validation Based Protocols With Simultaneous Hyper-Parameter Optimization". International Journal on Artificial Intelligence Tools, vol.XX no.X:1-30, World Scientific Publishing Company, 2015.

12. Gaspar, Paulo.,Carbonell, Jaime., Oliveira, Jose Luis., "On The Parameter Optimization Of Support Vector Machines For Binary Classification”. Journal of Integrative Bioinformatics, 9(3):201, 2012.

13. Syarif, Iwan.,Prugel-Bernnett, Adam., Wills, Gary., "Svm Parameter Optimization Using Grid Search And Genetic Algorithm To Improve Classification Performance”, Telkomnika Journal, 14(4):1502-1509, 2016. 